\title{
The LIM-Homeobox Gene Islet-1 Is Required for the Development of Restricted Forebrain Cholinergic Neurons
}

\author{
Yasser Elshatory ${ }^{1,2,3}$ and Lin Gan ${ }^{2,3}$ \\ ${ }^{1}$ Medical Scientist Training Program, ${ }^{2}$ Interdepartmental Graduate Program in Neuroscience, ${ }^{3}$ Department of Ophthalmology, University of Rochester \\ School of Medicine and Dentistry, Rochester, New York 14642
}

\begin{abstract}
Forebrain cholinergic neurons modulate complex mammalian behaviors such as reward-related learning and cognitive functions. Although their dysfunction is implicated in various psychiatric and neurodegenerative diseases, the factors governing cholinergic neuron differentiation and diversity are mostly unknown. We tested the role of the LIM-homeobox gene Isl1 in the development of forebrain cholinergic neurons by conditionally deleting Isl1 using a Six3-cre transgene. A depletion of cholinergic interneurons in the dorsal and ventral striatum, and cholinergic projection neurons in the nucleus basalis is observed and is ascribed to an early and persistent defect in cholinergic neuron differentiation. Notably, cholinergic innervation to the neocortex is abolished, whereas that to the hippocampus is unaltered. The unique pattern of cholinergic hypoinnervation encountered is supported by the presence of cholinergic projection neurons in the medial septum, the magnocellular preoptic area, and the substantia innominata. Together, these results demonstrate the requirement for Isl1 in the development of restricted telencephalic cholinergic neurons and link the development of cholinergic neurons in anatomically disparate sites to Isl1 function.
\end{abstract}

Key words: transcription factor; cholinergic neuron; differentiation; forebrain; basal forebrain; basalis; striatum

\section{Introduction}

Neurotransmitter identity is a fundamental characteristic of the nervous system, yet the genetic determinants of neurotransmitter selection are incompletely understood. Cholinergic neurons, for example, are found in discrete locations along the neuraxis and possess a striking level of anatomic heterogeneity (Woolf, 1991). Some are local circuit neurons, modulating reward-related learning in the striatum (Kitabatake et al., 2003), whereas others are projection neurons, targeting cortical and subcortical structures (Johnston et al., 1979; Lehmann et al., 1980; Woolf et al., 1983). Yet little is known about the genetic mechanisms underlying cholinergic neuron diversity.

Forebrain cholinergic neurons have demanded considerable attention, because disruption in subsets of these neurons has been implicated in the cognitive dysfunction associated with $\mathrm{Alz}$ heimer's disease (Davies and Maloney, 1976; Whitehouse et al., 1981). Forebrain cholinergic projection neurons are found in the medial septum (MS) (Ch1), the vertical (Ch2) and horizontal limbs (Ch3) of the nucleus of the diagonal band, and the basal magnocellular complex (Ch4) (Mesulam et al., 1983). Ch4 includes cholinergic neurons in the ventral pallidum, the nucleus

Received Dec. 27, 2007; revised Feb. 6, 2008; accepted Feb. 14, 2008.

This work was supported by National Institutes of Health Grant EY013426, a Research to Prevent Blindness (RPB) medical student fellowship, an Agnes M. and George Messersmith fellowship, and an RPB grant to the Department of Ophthalmology. Thanks to Drs. Yashuhide Furuta (Six3-cre mouse), Louis Reichardt (TrkA antibody), and Kuo-Fen Lee (Ngfr in situ probe). Thanks also to Dr. John Olschowka and Wei Pan for constructive discussions and technical assistance.

Correspondence should be addressed to Lin Gan, 601 Elmwood Avenue, Box 645, Rochester, NY 14642. E-mail: lin_gan@urmc.rochester.edu.

DOI:10.1523/JNEUROSCI.5730-07.2008

Copyright $\odot 2008$ Society for Neuroscience $\quad$ 0270-6474/08/283291-07\$15.00/0 basalis, ansa lenticularis, substantia innominata (SI), and the magnocellular preoptic nucleus. These designations correspond approximately to the axonal trajectories of the groups; Ch1 and Ch2 provide major innervation to the hippocampus; Ch3, the olfactory bulb; and Ch4, the neocortex (Mesulam et al., 1983).

Fate-mapping studies have suggested that cholinergic neurons emerge primarily from the ventral-most regions of the developing telencephalon in the anterior entopenduncular/preoptic area (AEP/POa) (Marin et al., 2000). Analysis of loss-offunction mutants for $N k \times 2-1, A s c l 1, D l \times 1 / 2$, and Arx reveal varying degrees of cholinergic marker loss, but a corresponding disruption of GABAergic interneurons suggests a broader role for these factors in basal telencephalic development (Sussel et al., 1999; Marin et al., 2000; Kitamura et al., 2002; Cobos et al., 2005; Colombo et al., 2007). Two genes downstream of Nkx2-1, Lhx6 and $L h x 8$ are required for forebrain GABAergic and cholinergic neuron development, respectively (Zhao et al., 2003; Alifragis et al., 2004; Mori et al., 2004; Liodis et al., 2007). But Lhx8independent forebrain cholinergic neurons exist, and Xenopus Lhx 8 overexpression in mouse subpallial neurons is not sufficient to expand cholinergic fates (Bachy and Retaux, 2006). Thus, other factors in addition to Lhx8 may be necessary for the differentiation of cholinergic neurons. LIM-homeodomain factors also function coordinately to instruct cell fate (Thor et al., 1999). The requirement for the LIM-homeobox gene Isl1 in spinal motor neuron and starburst amacrine differentiation (Pfaff et al., 1996; Elshatory et al., 2007b), both cholinergic neurons, makes Isl1 a candidate for regulating forebrain cholinergic differentiation. The expression of Isl1 in developing rat forebrain (Wang and Liu, 2001), the later restriction to subsets of cholinergic neurons (Wang and Liu, 2001), and the downregulation of Isl1 in 
Lhx8-null forebrains (Zhao et al., 2003) further support a role for Isl1 in forebrain cholinergic development.

Here, we present data using Isll conditional knock-outs showing the regulation by Isl1 of restricted populations of telencephalic cholinergic neurons that overlap with the pool of cholinergic neurons disrupted in Lhx8-null mice. The data provide evidence that forebrain cholinergic differentiation depends on the function of multiple LIM-homeobox genes, which may act hierarchically or coordinately to promote the differentiation of different subsets of cholinergic neurons.

\section{Materials and Methods}

Animals. The generation of and genotyping for the Isl1 ${ }^{\text {lac } Z}$ knock-in and floxed Isl 1 mice, Islf $f^{f l /+}$, has been described previously (Elshatory et al., 2007a,b). The Six3-cre transgenic line used in the present study has also been reported previously (Furuta et al., 2000). All animal procedures described herein were approved by the University Committee of Animal Resources at the University of Rochester.

For the purposes of staging embryos, noon of the day a vaginal plug was detected was taken to be embryonic day 0.5 (E0.5). Embryos were anesthetized on ice and immersion fixed for $3 \mathrm{~h}$ in $4 \%$ paraformaldehyde (PFA) at $4^{\circ} \mathrm{C}$, washed several times in PBS, and then cryoprotected in $30 \%$ sucrose in PBS before rapid freezing in OCT compound (TissueTek). To fix brains from postnatal day 0 (P0) pups and 3-week-old mice, animals were anesthetized, perfused transcardially with PBS, followed by 10 or $60 \mathrm{ml}$ of $4 \%$ PFA in PBS, pH 7.3. Brains were removed subsequently and postfixed overnight at $4^{\circ} \mathrm{C}$ in the same fixative, and then cryoprotected and frozen as described for embryos.

Immunohistochemistry, cell counts, and statistics. The $50 \mu \mathrm{m}$ freefloating frozen sections were stained using either Vectastain ABC kits (Vector Laboratories, Southfield, MI) or indirect immunofluorescence. For immunoperoxidase labeling, sections were blocked for $1 \mathrm{~h}$ using $\mathrm{PBS} / 10 \%$ normal horse serum and $0.3 \%$ Triton X-100 for P0 tissue or $0.4 \%$ Triton X-100 for adult tissue. Primary antibodies were diluted in PBS containing 3\% horse serum and the same detergent for at least $60 \mathrm{~h}$ at $4^{\circ} \mathrm{C}$. Endogenous peroxidase activity was quenched in $10 \%$ methanol and $0.1 \%$ hydrogen peroxide in PBS for $15 \mathrm{~min}$ at room temperature. Antibodies presented here were used as follows: rabbit anti-calretinin (1:1000; PC254L; Calbiochem, La Jolla, CA), goat anti-ChAT (1:200; AB144P; Millipore, Bellerica, MA), rabbit anti-dopamine- and cAMPregulated phosphoprotein of $32 \mathrm{kDa}$ (DARPP-32) (1:1000; AB1656; Millipore), mouse anti-Isl1/2 (1:200; catalog \#39.4D5; Developmental Studies Hybridoma Bank, Iowa City, IA), rabbit anti-neuropeptide Y (NPY) (1:3000; Immunostar, Hudson, WI), mouse anti-parvalbumin (1:200; Sigma, St. Louis, MO), rabbit anti-phosphorylated histone H3 (1:400; Santa Cruz Biotech, Santa Cruz, CA), and rabbit anti-TrkA (1:500; courtesy of Dr. Louis Reichardt, University of California, San Francisco, San Francisco, CA). Biotinylated secondary antibodies were applied for $2 \mathrm{~h}$ at room temperature in the same diluent as the primary antibodies. Sections were developed using hydrogen peroxide substrate in a $0.05 \% \mathrm{DAB}$ solution. Cell counts were performed by counting at least five sections per animal, using external anatomical references as landmarks. Student's $t$ tests were used for all pairwise comparisons between Isl1 ${ }^{f l f l}$; Six3-cre and control littermates.

5-Bromo-4-chloro-3-indolyl- $\beta$-D-galactopyranoside staining, acetylcholinesterase histochemistry, and in situ hybridization. 5-Bromo-4-chloro3 -indolyl- $\beta$-D-galactopyranoside (X-gal) staining was performed on 30 $\mu \mathrm{m}$ free-floating sections, as described previously (Elshatory et al., 2007b). X-gal staining preceded immunohistochemistry in X-gal/immunohistochemistry double labeling. Acetylcholinesterase histochemistry was performed on $30 \mu \mathrm{m}$ free-floating frozen sections as described previously (Shaftel et al., 2007). In situ hybridization was carried out on 30 $\mu \mathrm{m}$ frozen sections as described previously (Elshatory et al., 2007b). Digoxygenin-labeled antisense probes were synthesized in vitro from $\mathrm{cD}$ NAs for $\operatorname{Lhx} 6, \operatorname{Lhx} 8$ (also $\operatorname{Lhx} 7$ ), and $N g f r$ (also p75; courtesy of Dr. Kuo-Fen Lee, University of California, San Diego, La Jolla, CA).

\section{Results}

\section{Expression of Isl1-lac Z in the mouse basal forebrain}

Isl1 expression has been detected in diverse cholinergic pools in the rat nervous system (Thor et al., 1991; Galli-Resta et al., 1997; Wang and Liu, 2001). In the adult mouse brain, colocalization of Isl1-lacZ expression with markers of cholinergic neurons revealed expression of Isl1-lac $Z$ in cholinergic interneurons of the dorsal striatum (Fig. $1 A$ ) and ventral striatum (Fig. $1 B$ ), but also in cholinergic projection neurons in the nucleus basalis (Fig. 1C), and fewer cholinergic projection neurons in the magnocellular preoptic area (MCPO) (Fig. 1D) and MS (Fig. 1E). The percentage of colocalization of Isl1-lacZ and ChAT varied across the groups, with the highest percentages being encountered in the caudate-putamen and nucleus basalis (average $\pm \mathrm{SD}, n=3$ : $95.4 \pm 0.6$ and $86.9 \pm 7.3 \%$, respectively). The lowest percentages of colocalization were encountered in the MCPO/SI and Ch1-2 (average $\pm \mathrm{SD}, n=3: 35.6 \pm 14.4$ and $24.6 \pm 6.1 \%$, respectively). However, expression was not detected in striatal projection neurons immunoreactive for DARPP-32 (Ppplr1b) (Fig. 1F) or GABAergic interneurons in the striatum immunoreactive for NPY (Fig. $1 G$ ), parvalbumin (Fig. $1 H$ ), or calretinin (calbindin 2) (Fig. 1I). The colocalization of the recombination pattern of Six3-cre with cholinergic and striatal interneuron markers was gauged to determine the appropriateness of its use to study the function of Isl 1 in the forebrain. Most cholinergic interneurons were positive for the lineage of Six3-cre (Fig. 1J, black arrowhead), although a minor population of reporter-negative cholinergic interneurons was encountered (Fig. $1 \mathrm{~J}$, white arrowhead). Broader Six3-Cre-mediated recombination was encountered in the nucleus basalis and medial septum, encompassing most cholinergic projection neurons in these regions (Fig. $1 K, L$, black arrowheads). The percentages of colocalization of Six3-cre-R26R and ChAT indicate that Six 3 -cre recombination occurs in the majority of forebrain cholinergic neurons. For example, in the caudate-putamen, $78.1 \pm 5.9 \%$ of $\mathrm{ChAT}^{+}$interneurons were Six3-cre-R26R ${ }^{+}, 89.2 \pm 3.5 \%$ of cholinergic neurons in the nucleus basalis were Six 3 -cre-R26R ${ }^{+}$, and $91.9 \pm 3.4 \%$ were positive in Ch1-2 neurons (averages $\pm \mathrm{SD} ; n=3$ ). $\mathrm{NPY}^{+}$and parvalbumin-positive striatal interneurons similarly expressed the reporter (Fig. $1 \mathrm{M}, \mathrm{N}$, black arrowheads), whereas calretininpositive striatal interneurons were routinely negative for reporter expression (Fig. 1O, white arrowheads). Examination of the developing forebrain at E13.5 revealed Isl1 expression in cells negative for phosphorylated histone $\mathrm{H} 3$ expression, an M-phase cell cycle marker, in the subventricular zone of the lateral ganglionic eminence (LGE) and the AEP/POa, as well as scattered cells in between (Fig. $1 P$ ). The ventral-most expression of Isl1 in postmitotic neurons of the AEP/POa is suggestive of a role in the differentiation of neurons emanating from this region, such as cholinergic neurons. Analysis of $I s l l^{\text {flffl }}$; Six3-cre mice revealed a depletion of Isl1 expression in the AEP/POa (Fig. 1Q, arrowheads), but its maintenance more dorsolaterally in the LGE (Fig. $1 Q$, bracket). This pattern of Isl 1 deletion is consistent with the greater efficiency of Six3-cre recombination in the AEP/POa compared with the LGE (supplemental Fig. 1, available at www. jneurosci.org as supplemental material). More anteriorly, Isl1 expression in the septal anlagen is also abolished (data not shown).

\section{Marked reduction in cholinergic but not GABAergic interneurons in striata of adult Isl1 conditional knock-outs}

The expression of interneuron striatal markers was assessed in Islf ${ }^{f l f f}$; Six 3-cre animals compared with their littermate controls at 3 weeks of age. Labeling for the acetylcholine synthetic enzyme 


\section{ChAT/Is/1-lacZ}

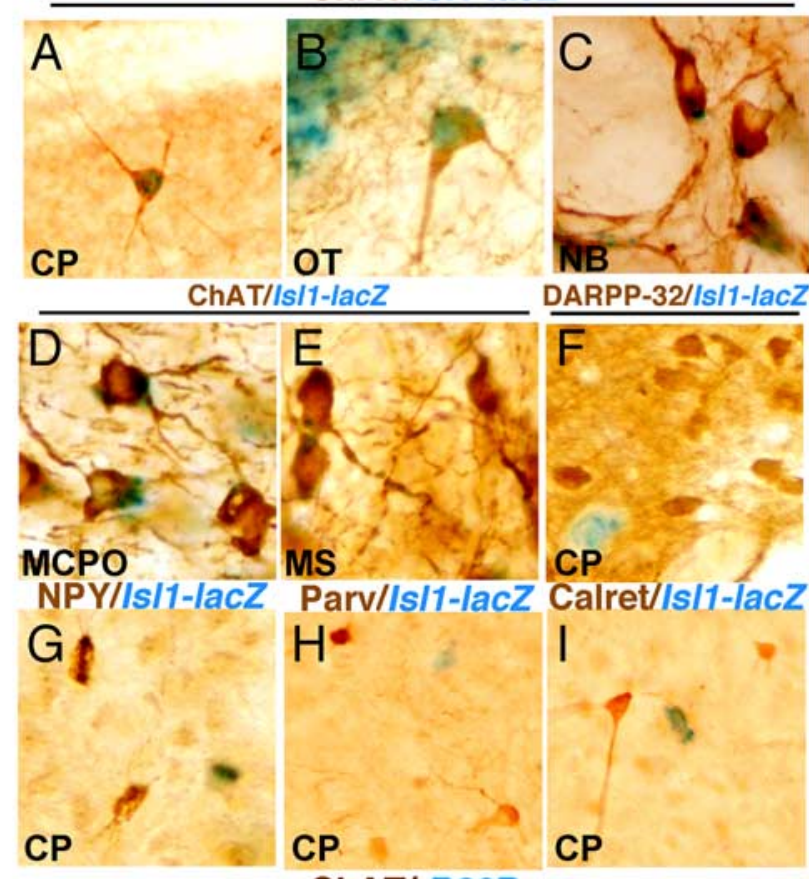

ChAT/ R26R
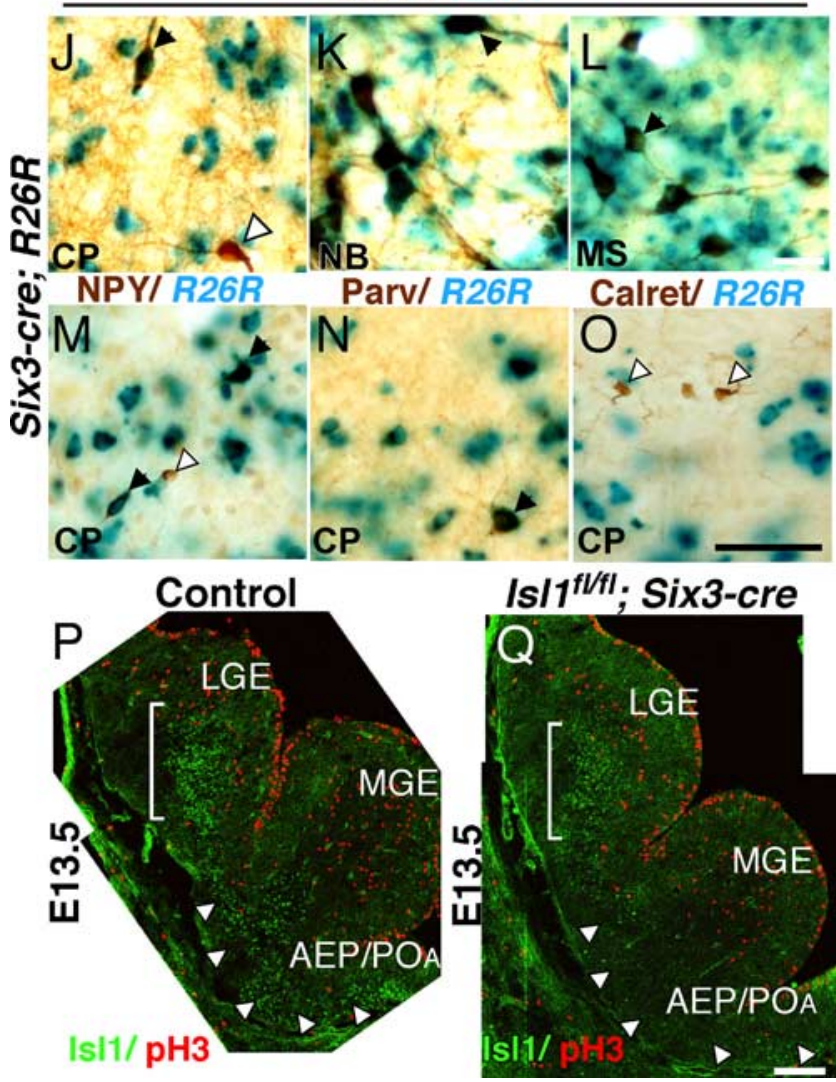

Figure 1. Isl1-lacZ expression in restricted forebrain cholinergic neurons. A-I, Light micrographs of X-gal-stained tissue from 3-week-old $/ s / 7^{\text {lacz/+ }}$ mice double labeled for cholinergic and striatal markers demonstrating colocalization of Is/1-lacZ expression with the cholinergic marker ChAT in the caudate-putamen (CP) $(\boldsymbol{A})$, olfactory tubercle $(0 \mathrm{OT})(\boldsymbol{B})$, and nucleus basalis $(\mathrm{NB})(\boldsymbol{C})$, with varying colocalization in the MCPO $(\boldsymbol{D})$ and MS $(\boldsymbol{E})$. Colocalization was not encountered in DARPP $-32^{+}$striatal projection neurons $(\boldsymbol{F})$, or other interneuron subtypes in the $\mathrm{CP}_{\text {, including NPY }}{ }^{+}(\boldsymbol{G})$, parvalbumin-positive $(\boldsymbol{H})$, or calretinin-positive $(\boldsymbol{I})$ interneurons. $\boldsymbol{J}-\mathbf{0}$, Light micrographs of X-gal-stained tissue from 3-week-old Six3-cre; $R 26 R$ mice demonstrating colocalization of the recombination pattern of Six3-cre with ChAT in the $C P(\boldsymbol{J}), \mathrm{NB}(\boldsymbol{K})$, and MS (L). Colocalization was also demonstrated in $\mathrm{NPY}^{+}(\boldsymbol{M})$ and parvalbumin-positive $(\boldsymbol{N})$ but not
ChAT demonstrated an $86 \%$ reduction in cholinergic interneuron marker expression in the caudate-putamen of Isll conditional knock-outs (Fig. $2 A, B$ ) (average number of $\mathrm{ChAT}^{+}$interneurons \pm SD: controls, $266 \pm 25, n=5$; Isll ${ }^{\text {flffl }}$; Six3-cre, $36 \pm$ $20, n=5 ; p<0.0002$, Student's $t$ test). This defect is likely cell-autonomous, because residual Isll expression in Isll conditional knock-outs is sufficient to confer ChAT expression in the caudate-putamen (supplemental Fig. 2, available at www. jneurosci.org as supplemental material). Cholinergic interneurons in the olfactory tubercle and nucleus accumbens were also markedly reduced (data not shown). The other predominant neurotransmitter phenotype of striatal interneurons is GABAergic, and this group of neurons are further subdivided into subtypes based on the expression of calcium-binding proteins (calretinin, parvalbumin) and neuropeptides (NPY, somatostatin) (Kawaguchi, 1993; Figueredo-Cardenas et al., 1996a,b). No significant difference in the numbers of calretinin, parvalbumin, or NPY-positive interneurons was detected in the caudate-putamen of Isll conditional knock-outs compared with controls (Fig. 2C$H$ ). The observation that calretinin-positive interneurons are negative for the lineage of Six3-cre in the caudate-putamen (Fig. $1 O$ ) indicates a possibility still that Isll could regulate the development of this cell type. Using anti-DARPP-32 immunolabeling to reveal the area occupied by striatal projection neurons revealed a slight but significant $26 \%$ reduction in Isl1 conditional knockouts (supplemental Fig. 3, available at www.jneurosci.org as supplemental material). Together, Isll deletion leads to a profound disruption of cholinergic interneurons in the dorsal and ventral striatum, and does so without disrupting the expression of other interneuron phenotypes significantly.

\section{Severe reduction of cholinergic projection neurons in the} nucleus basalis of adult Isll conditional knock-outs

The expression of ChAT was assessed in Isll flffl ; Six3-cre animals and control littermates at 3 weeks of age in various subcortical structures in which cholinergic projection neurons are located. The numbers of cholinergic neurons in the nucleus basalis of Isl1 conditional knock-outs was reduced by $83 \%$ compared with controls (Fig. 3A-D) (average number of $\mathrm{ChAT}^{+}$neurons $\pm \mathrm{SD}$ : controls, $67 \pm 9, n=5 ;$ Isl1 ${ }^{f l f l} ;$;ix 3 -cre, $11 \pm 7, n=5 ; p<0.0005$, Student's $t$ test). Because cholinergic projection neurons in this nucleus provide the predominant cholinergic innervation to the neocortex, acetylcholinesterase staining of the neocortex of Isll conditional knock-outs and controls was assessed. Isl1 ${ }^{f l f l}$; Six3cre mice demonstrated a substantial loss of neocortical cholinergic innervation (Fig. $3 E, F$ ). Cholinergic projection neurons in $\mathrm{Ch} 1$ and $\mathrm{Ch} 2$, as well as other Ch4 subdivisions (Fig. 3G-J) were not as severely affected as in $L h \times 8$-null mice, which display significant reductions in Ch1 and Ch2 (56-77\%), as well as severe reductions across Ch4 subdivisions (i.e., MCPO/SI, 82-96\%) (Zhao et al., 2003; Mori et al., 2004; Fragkouli et al., 2005). In Isl1 conditional knock-outs, a $27 \%$ reduction in $\mathrm{ChAT}^{+} \mathrm{MCPO} / \mathrm{SI}-$ Ch4 neurons was observed compared with controls (average number of $\mathrm{ChAT}^{+} \mathrm{MCPO} / \mathrm{SI}-\mathrm{Ch} 4$ neurons \pm SD: controls, $231 \pm 40, n=5$; Isl1 ${ }^{f l / f l}$; Six3-cre, $170 \pm 37, n=5 ; p<0.05$,

calretinin-positive (0) interneurons. $\mathbf{P}-\mathbf{Q}$, Confocal micrographs of $|s| 1$ and $\mathrm{pH} 3-$ immunolabeling at E13.5 demonstrating exclusion of Is 11 from $\mathrm{pH} 3{ }^{+}$mitotic cells in the LGE subventricular zone (SVZ) as well as the AEP/P0a. Six3-cre-mediated recombination of floxed Is 11 resulted in depletion of Is 11 immunolabeling in the AEP/POa ( $\mathbf{Q}$, arrowheads), but residual expression in the $L G E(\boldsymbol{Q}$, brackets). MGE, Medial ganglionic eminence. Scale bars: (in $\mathbf{0}) \mathbf{A}-\mathbf{0}, 20$ $\mu \mathrm{m}$; (in $\mathbf{Q}) \mathbf{P}, \mathbf{Q}, 100 \mu \mathrm{m}$ 


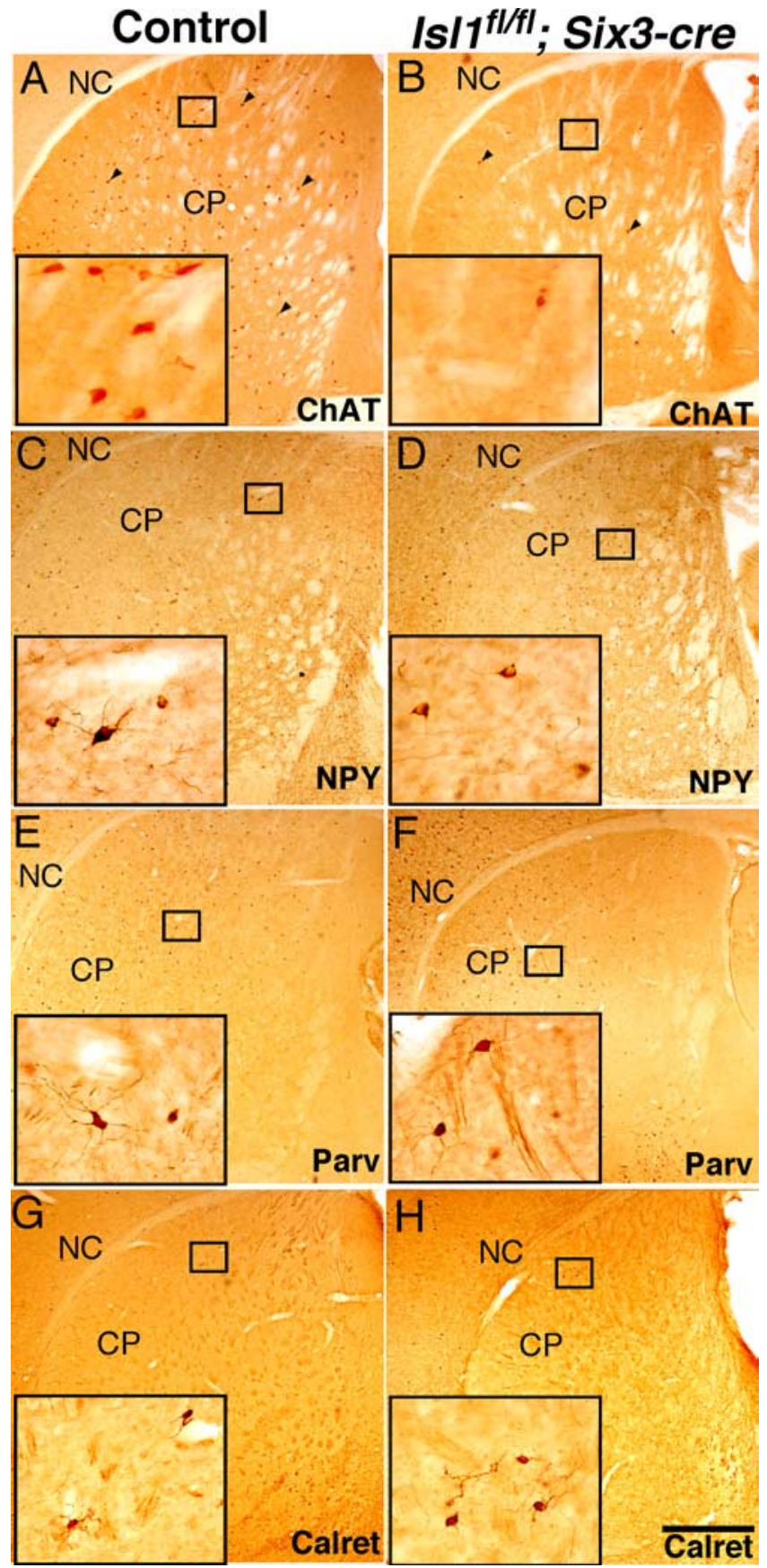

Figure 2. Cholinergic interneurons are preferentially reduced in 3-week-old $\mid s / 1$ conditional knock-outs. $\boldsymbol{A}-\boldsymbol{H}$, Light micrographs of striatal interneuron marker expression demonstrating a marked reduction in the cholinergic interneuron marker $C h A T$ in $/ s / 1$ conditional knock-outs ( $\boldsymbol{B}$, arrowheads) compared with controls ( $\boldsymbol{A}$, arrowheads; average number of $\mathrm{ChAT}^{+}$interneurons $\pm \mathrm{SD}$ : controls, $266 \pm 25, n=5 ; / s / 7^{A / f / f} ;$ Six 3 -cre, $36 \pm 20, n=5 ; p<0.0002$, Student's $t$ test). NPY ${ }^{+}$ interneurons were not significantly different between $/ s / 1$ conditional knock-outs ( $\boldsymbol{D}$ ) and controls ( $\boldsymbol{C}$; average number of $\mathrm{NPY}^{+}{ }^{+}$interneurons $\pm \mathrm{SD}$ : controls, $232 \pm 24, n=5 ; / \mathrm{s} / 7^{t / / f / f} ; \mathrm{Six} 3-\mathrm{cre}, 230 \pm 33$, $n=4 ; p \leq 0.93$, Student's $t$ test). Parvalbumin-positive interneurons $(\boldsymbol{E}, \boldsymbol{F})$ also showed no significant difference between groups (average number of parvalbumin-positive interneurons \pm SD: controls, $264 \pm 80, n=5 ; \mathrm{Is} / 1^{f / f / f} ;$ Six3-cre, $206 \pm 46, n=5 ; p \leq 0.20$, Student's $t$ test). Similarly, calretinin-positive interneurons $(\boldsymbol{G}, \boldsymbol{H})$ were not significantly different (average number of calretininpositive interneurons $\pm \mathrm{SD}$ : controls, $15 \pm 3, n=5 ; \mathrm{ISI} / 7^{f / f f /} ; \mathrm{Six} 3-\mathrm{cre}, 18 \pm 6, n=5 ; p \leq 0.35$, Student's $t$ test). NC, Neocortex. Scale bar, $250 \mu \mathrm{m}$.

Student's $t$ test), whereas $\mathrm{ChAT}^{+} \mathrm{Ch} 1-2$ neurons were not significantly affected (average number of $\mathrm{ChAT}^{+} \mathrm{Ch} 1-2$ neurons \pm SD: controls, $142 \pm 33, n=4$; Isl1 ${ }^{f l f l}$; Six3-cre, $156 \pm 14, n=3$;
NS, Student's $t$ test). Correspondingly, acetylcholinesterase staining of hippocampal tissue, which derives mostly from Ch1 and Ch2 (Mesulam et al., 1983) was preserved in Isl1 conditional knock-outs (Fig. $3 K-L$ ).

\section{Early impairment of forebrain cholinergic differentiation in} Isl 1 conditional knock-outs

The expression of multiple markers of cholinergic neurons was assessed at P0 in $I s l 1^{f l f l}$; Six3-cre and control littermates to assess earlier differentiation of cholinergic neurons after Isl1 disruption. ChAT expression revealed an early disruption of cholinergic interneurons in the caudate-putamen and cholinergic projection neurons in the nucleus basalis compared with controls (Fig. $4 A, B)$. Ngfr (also p75) mRNA expression confirmed the early disruption in cholinergic neuron differentiation in the nucleus basalis (Fig. 4C,D), whereas cholinergic neurons in the magnocellular preoptic area appeared to express $N g f r$ mRNA normally (Fig. 4E,F). TrkA immunohistochemistry, which identifies basal telencephalic cholinergic neurons (Sussel et al., 1999), reaffirmed the defect observed in cholinergic interneuron differentiation of the caudate-putamen (Fig. 4G,H) and cholinergic projection neuron differentiation in the nucleus basalis (Fig. 4I,J), because TrkA expression was markedly reduced. Although expression levels of $L h x 8$ mRNA in the caudate-putamen appeared to be reduced in $I s l 1$ conditional knock-outs (Fig. $4 K, L$ ), it was still present, and more numerous than could be accounted for by residual cholinergic interneurons (Fig. $4 B, H$ ). This suggests that early markers of cholinergic development persist, whereas acquisition of mature cholinergic markers is disrupted (Fig. 4M,N). This is also supported by persistent $I s l 1-$ lac $^{+}$cells in the caudate-putamen of Isl1 conditional knock-outs, although a broad overexpression of Isl1-lacZ expression in conditional knock-outs obscures clear identification of putative cholinergic interneurons (supplemental Fig. 4, available at www.jneurosci.org as supplemental material). More caudally, $L h x 8$ mRNA expression is maintained in the ansa lenticularis (Fig. 4O,P), which represents a structure also containing cholinergic projection neurons that closely apposes the nucleus basalis. Cholinergic projection neurons here are also decreased in Isl1 conditional knock-outs (data not shown), and may represent persisting neurons expressing early markers of cholinergic neurons but failing to differentiate. The close juxtaposition of the ansa lenticularis to the globus pallidus, a structure in which $\operatorname{Lh} x 8$ is also expressed (Zhao et al., 2003), makes it difficult to conclude definitively that early markers of cholinergic projection neurons persist in Isl1 conditional knock-outs. Lhx6 mRNA expression was not apparently affected in the neocortex (Fig. 4Q, $R$, asterisks) or caudate-putamen (Fig. $4 Q, R$, black arrowheads), suggesting the specification of GABAergic interneurons is unaffected in $I s l l^{f l f l}$; Six3-cre animals.

\section{Discussion}

Isl1 deletion leads to a profound disruption of cholinergic interneurons in the dorsal and ventral striatum, and appears to do so without disrupting the expression of other interneuron phenotypes significantly. Although the reduction in the area occupied by DARPP $-32{ }^{+}$neurons might arise from an intrinsic role for Isl 1 in DARPP $-32^{+}$neurons as postulated (supplemental Fig. 3, available at www.jneurosci.org as supplemental material) (Wang and Liu, 2001; Stenman et al., 2003), it may also be a consequence of the reported reduction in body size in Isll conditional knockouts (Elshatory et al., 2007a). If merely a reflection of reduced body size, the reduction in striatal area should generalize to reductions in other brain structures, such as the thickness of the 


\section{Control}
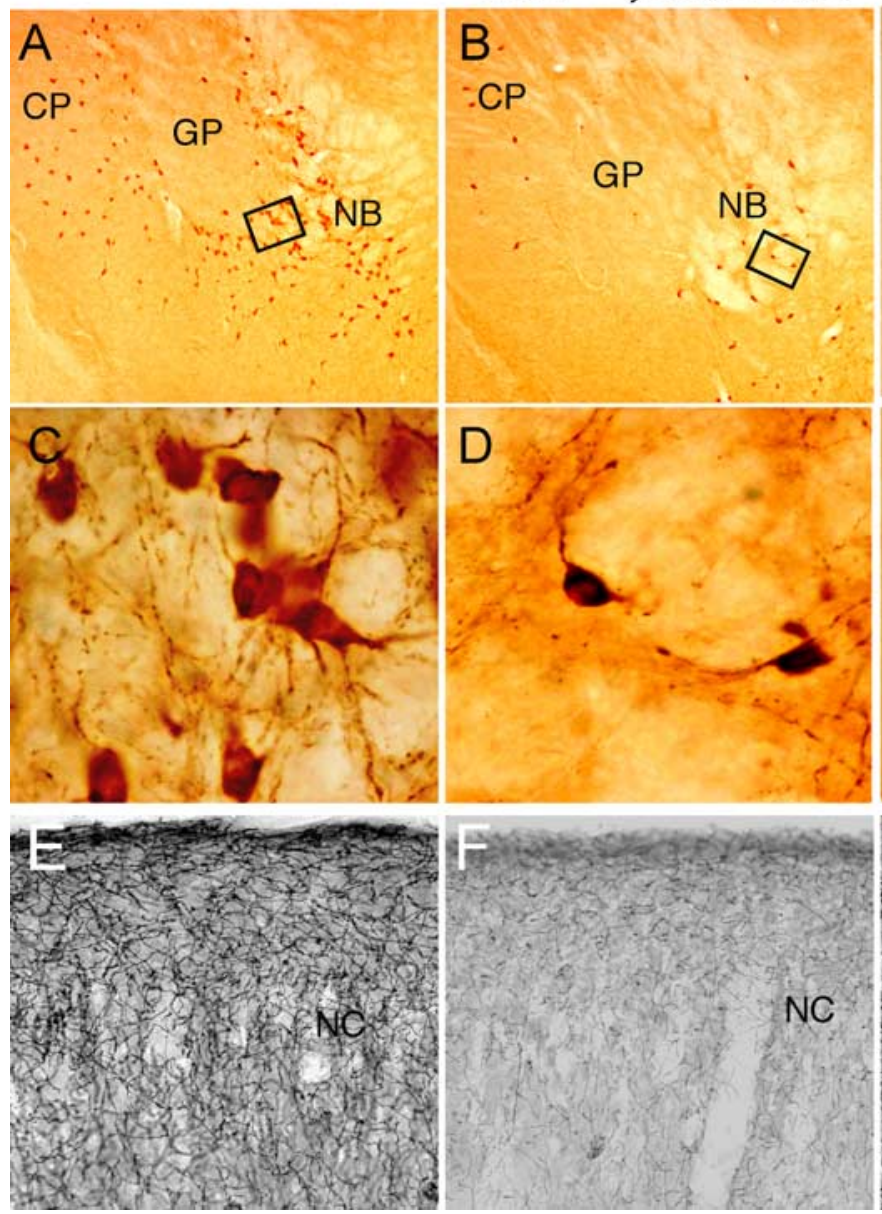

Control
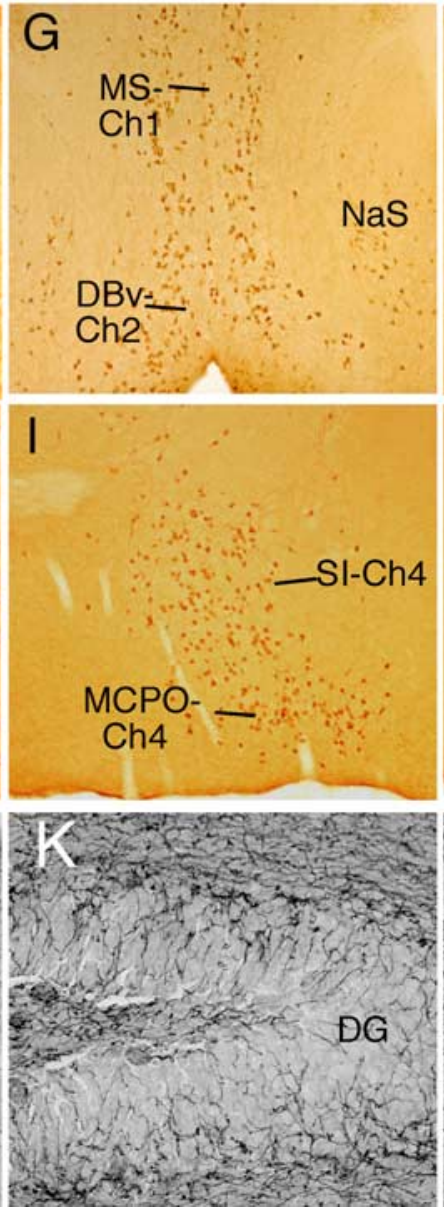

$|s| 1^{f l / f I} ;$ Six3-cre
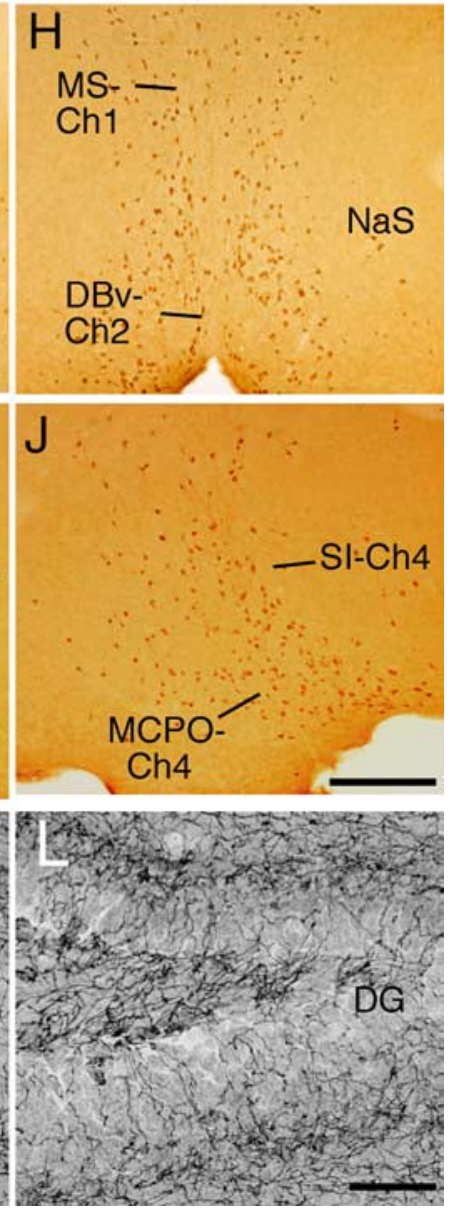

Figure 3. Restricted reduction of cortically projecting cholinergic neurons of the nucleus basalis in the $/ s / 1$ conditional knock-out. $A-D$, Light micrographs of anti-ChAT staining in the nucleus basalis (NB) of control and $/ s / 1$ conditional knock-outs at 3 weeks of age demonstrating a loss of the majority of ChAT ${ }^{+}$cells in the NB.E, $F$, Light micrographs of acetylcholinesterase staining at the neocortical surface in coronal sections of control $(\boldsymbol{E})$ and $/ s / 1$ conditional knock-out $(\boldsymbol{F})$ brains showing reduced acetylcholinesterase staining in the cortex. $\mathbf{G}-J$, Light micrographs of anti-ChAT staining of additional forebrain cholinergic populations. Anti-ChAT expression in the MS and vertical limb of the diagonal band (DBv) is not markedly reduced in conditional knock-outs ( $\boldsymbol{H}$ ) compared with controls (G). Expression of ChAT ${ }^{+}$interneurons in the nucleus accumbens shell (NaS), however, is reduced (compare $\boldsymbol{G}, \boldsymbol{H}$ ). Anti-ChAT staining of the MCP0 and adjacent $\mathrm{Sl}$ is not substantially reduced in $/ s / 1$ conditional knock-outs $(J)$ compared with controls $(I)$. Additional acetylcholinesterase staining reveals no clear disruption in cholinergic innervation to the hippocampus in conditional knock-outs $(\boldsymbol{L})$ versus controls $(\boldsymbol{K})$. GP, Globus pallidus; NC, neocortex; DG, dentate gyrus. Scale bars: (in $\boldsymbol{J}) \boldsymbol{A}, \boldsymbol{B}, \boldsymbol{G}-\boldsymbol{J}, 250 \mu \mathrm{m}$; (in $\boldsymbol{L}) \boldsymbol{E}, \boldsymbol{F}, \boldsymbol{K}, \boldsymbol{L}, 20 \mu \mathrm{m}$.

cerebral cortex. However, cortical thickness was not significantly reduced in Isll conditional knock-outs, suggesting that Isl1 likely functions in the development of striatal projection neurons (average cortical thickness $\pm \mathrm{SD}$ : controls, $1.21 \pm 0.09 \mathrm{~mm}, n=4$; Isl $1^{f l f f}$; Six3-cre, $1.24 \pm 0.06 \mathrm{~mm}, n=4$; NS, Student's $t$ test). This finding is more compelling given the limited recombination of Six3-cre in LGE-derived cells (supplemental Fig. 1, available at www.jneurosci.org as supplemental material) and suggests more efficient deletion of Isl 1 in this region using Nestin-cre may reveal a more widespread role for Isl1 in striatal development. The reduced area of the caudate-putamen, however, cannot account for the degree of cholinergic interneuron loss that was observed. The cholinergic neuron loss in Isl 1 conditional knock-outs confirms that multiple LIM-homeodomain proteins regulate forebrain cholinergic fate, and establish a scenario in which Isl1, Lhx8, and Lhx6 function in the context of dense transcriptional codes (Flames et al., 2007) to instruct the differentiation of diverse GABAergic and cholinergic neuronal populations. There is a possibility that the remaining cholinergic projection neurons are maintained in Isl 1 conditional knock-outs because Isll is not adequately deleted, but the widespread recombination of Six3-cre in basal forebrain nuclei (Fig. $1 K, L$ ), and the robust deletion of Isl1 in the $\mathrm{AEP} / \mathrm{POa}$ and septal anlagen suggest otherwise (Fig. 1Q) (data not shown). Isl1 conditional knock-outs were found to have varying degrees of cholinergic neuron reduction across forebrain cholinergic groups, but the degree of reduction encountered corresponded approximately to the degree of colocalization of Isl1lac $Z$ and ChAT in these groups. That is, there was an $86 \%$ reduction in cholinergic interneurons in the caudate-putamen, an $83 \%$ reduction in cholinergic neurons in the nucleus basalis, a $27 \%$ reduction in the $\mathrm{MCPO} / \mathrm{SI}$, and no significant difference in Ch1-2 cholinergic neurons (Fig. 3). The high percentage of Six3cre recombination in Ch1-2, and the lack of significant reduction in this pool of cholinergic neurons in Isll conditional knock-outs (Fig. 3) suggest that the differentiation of these cells is mostly Isl1-independent.

Fate-mapping experiments have suggested that cholinergic neurons arise from the AEP/POa (Marin et al., 2000). Because Isl1 expression is more completely ablated in the AEP/POa than the LGE in Isl1 conditional knock-outs, and because this correlates with defects in cholinergic neuron differentiation, our findings support earlier assertions regarding the origins of cholinergic 


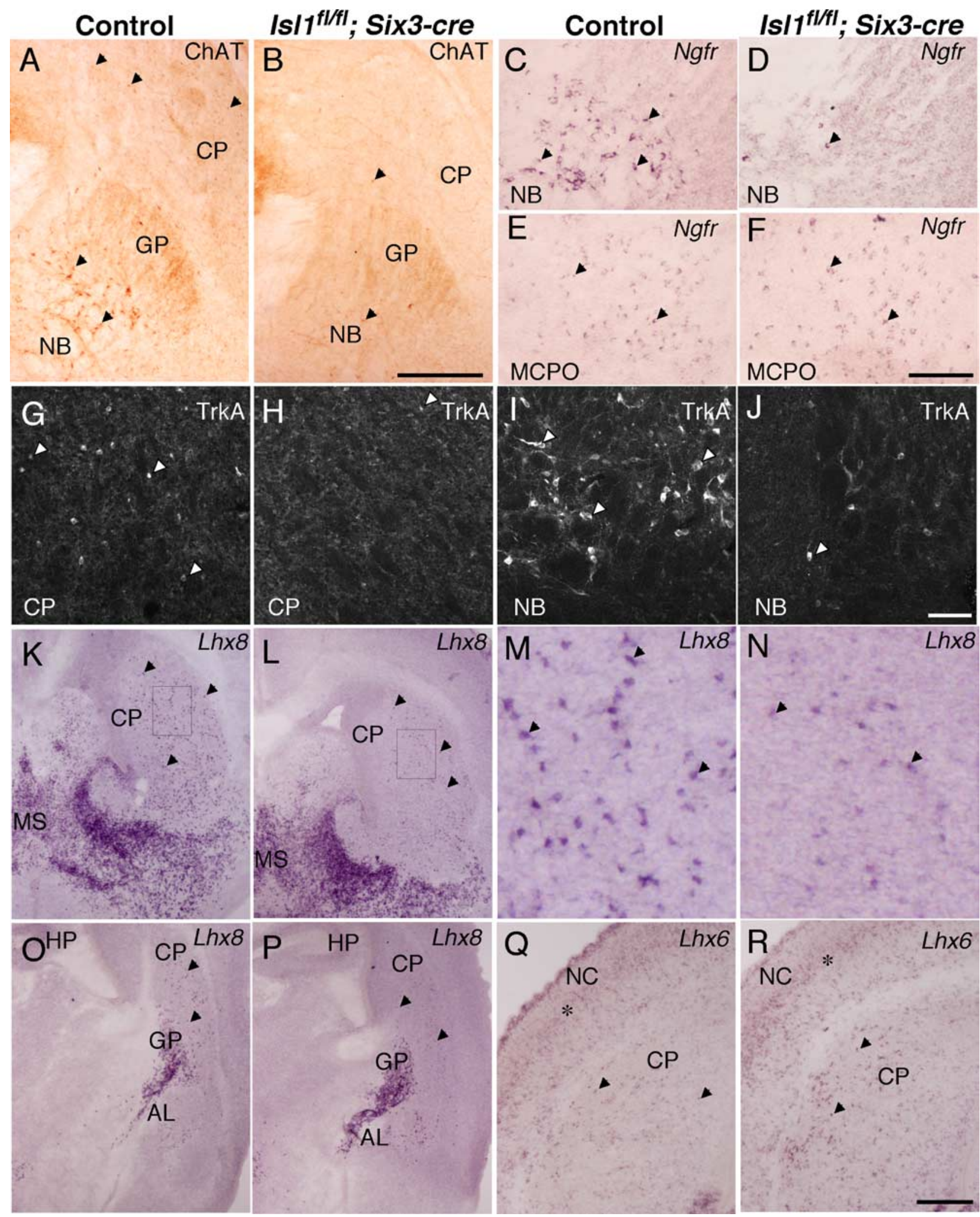

Figure 4. Subsets of cholinergic neurons fail to differentiate in the $/ s / 1$ conditional knock-out telencephalon at $P 0 . A, B$, Light micrographs of anti-ChAT staining at $P 0$ demonstrating its reduction in the nucleus basalis (NB) and caudate-putamen (CP) in knock-outs ( $\boldsymbol{B}$, arrowheads) versus controls ( $\boldsymbol{A}$, arrowheads). Ngfr mRNA expression is considerably reduced in the NB of PO knock-outs ( $\boldsymbol{D}$, arrowheads) versus controls ( $\boldsymbol{C}$, arrowheads), whereas expression in the MCPO of knock-outs ( $\boldsymbol{F}$, arrowheads) is not clearly disrupted compared with controls ( $\boldsymbol{E}$, arrowheads). Anti-TrkA immunofluorescence shows a reduction in TrkA ${ }^{+}$neurons in the $\mathrm{CP}$ of knock-outs ( $\boldsymbol{H}$, arrowhead) compared with controls ( $\boldsymbol{G}$, arrowheads) at P0, and a similar reduction in TrkA ${ }^{+}$neurons in the NB of knock-outs $(\boldsymbol{J}$, arrowheads) compared with controls $(\boldsymbol{I}$, arrowheads). Lhx8 mRNA expression reveals no clear reduction of $L h x 8$ expression in the MS, but a decrease in $(P$ expression levels in knock-outs $(\boldsymbol{L}$, arrowheads) versus controls ( $\boldsymbol{K}$, arrowheads). Higher magnification of $L h \times 8 \mathrm{mRNA}$ expression in the $(P$ demonstrates the presence of sustained $L h \times 8$ expression despite the lower expression levels in knock-outs ( $\boldsymbol{N}$, arrowheads) compared with controls ( $\boldsymbol{M}$, arrowheads). Lhx8 mRNA is also maintained in the globus pallidus (GP) and, putatively, the ansa lenticularis (AL) in conditional knock-outs ( $\boldsymbol{P}$, arrowheads) as in controls ( $\boldsymbol{O}$, arrowheads). Lhx6 mRNA expression is not clearly disrupted, both in the neocortex (NC) (asterisks) and CP (arrowheads) in conditional knock-outs ( $\boldsymbol{R}$, arrowheads) versus controls ( $\boldsymbol{Q}$, arrowheads). HP, Hippocampus. Scale bars: (in $\boldsymbol{B}) \boldsymbol{A}, \boldsymbol{B}, 250 \mu \mathrm{m}$; (in $\boldsymbol{F}$ ) $\mathbf{C}-\boldsymbol{F}, 100 \mu \mathrm{m}$; (in $\boldsymbol{J}) \boldsymbol{G}-\boldsymbol{J}, 50 \mu \mathrm{m}$; (in $\boldsymbol{R}) \boldsymbol{K}, \boldsymbol{L}, \mathbf{0}-\boldsymbol{R}, 500 \mu \mathrm{m}$. 
neurons. For subsets of forebrain cholinergic neurons in Isl1 conditional knock-outs, early markers of cholinergic development persist, whereas acquisition of mature cholinergic markers is disrupted (Fig. 4). This is also supported by persistent Isl1-lacZ ${ }^{+}$ cells in the caudate-putamen of Isll conditional knock-outs (supplemental Fig. 4, available at www.jneurosci.org as supplemental material). Together, Isll disruption seems to affect preferentially the differentiation of a subset of forebrain cholinergic neurons.

\section{References}

Alifragis P, Liapi A, Parnavelas JG (2004) Lhx6 regulates the migration of cortical interneurons from the ventral telencephalon but does not specify their GABA phenotype. J Neurosci 24:5643-5648.

Bachy I, Retaux S (2006) GABAergic specification in the basal forebrain is controlled by the LIM-hd factor Lhx7. Dev Biol 291:218-226.

Cobos I, Broccoli V, Rubenstein JL (2005) The vertebrate ortholog of Aristaless is regulated by Dlx genes in the developing forebrain. J Comp Neurol 483:292-303.

Colombo E, Collombat P, Colasante G, Bianchi M, Long J, Mansouri A, Rubenstein JL, Broccoli V (2007) Inactivation of Arx, the murine ortholog of the X-linked lissencephaly with ambiguous genitalia gene, leads to severe disorganization of the ventral telencephalon with impaired neuronal migration and differentiation. J Neurosci 27:4786-4798.

Davies P, Maloney AJ (1976) Selective loss of central cholinergic neurons in Alzheimer's disease. Lancet 2:1403.

Elshatory Y, Deng M, Xie X, Gan L (2007a) Expression of the LIMhomeodomain protein Isl1 in the developing and mature mouse retina. J Comp Neurol 503:182-197.

Elshatory Y, Everhart D, Deng M, Xie X, Barlow RB, Gan L (2007b) Islet-1 controls the differentiation of retinal bipolar and cholinergic amacrine cells. J Neurosci 27:12707-12720.

Figueredo-Cardenas G, Medina L, Reiner A (1996a) Calretinin is largely localized to a unique population of striatal interneurons in rats. Brain Res 709:145-150.

Figueredo-Cardenas G, Morello M, Sancesario G, Bernardi G, Reiner A (1996b) Colocalization of somatostatin, neuropeptide Y, neuronal nitric oxide synthase and NADPH-diaphorase in striatal interneurons in rats. Brain Res 735:317-324.

Flames N, Pla R, Gelman DM, Rubenstein JL, Puelles L, Marin O (2007) Delineation of multiple subpallial progenitor domains by the combinatorial expression of transcriptional codes. J Neurosci 27:9682-9695.

Fragkouli A, Hearn C, Errington M, Cooke S, Grigoriou M, Bliss T, Stylianopoulou F, Pachnis V (2005) Loss of forebrain cholinergic neurons and impairment in spatial learning and memory in LHX7-deficient mice. Eur J Neurosci 21:2923-2938.

Furuta Y, Lagutin O, Hogan BL, Oliver GC (2000) Retina- and ventral forebrain-specific Cre recombinase activity in transgenic mice. Genesis 26:130-132.

Galli-Resta L, Resta G, Tan SS, Reese BE (1997) Mosaics of islet-1expressing amacrine cells assembled by short-range cellular interactions. J Neurosci 17:7831-7838.

Johnston MV, McKinney M, Coyle JT (1979) Evidence for a cholinergic projection to neocortex from neurons in basal forebrain. Proc Natl Acad Sci USA 76:5392-5396.

Kawaguchi Y (1993) Physiological, morphological, and histochemical characterization of three classes of interneurons in rat neostriatum. J Neurosci 13:4908-4923.

Kitabatake Y, Hikida T, Watanabe D, Pastan I, Nakanishi S (2003) Impair- ment of reward-related learning by cholinergic cell ablation in the striatum. Proc Natl Acad Sci USA 100:7965-7970.

Kitamura K, Yanazawa M, Sugiyama N, Miura H, Iizuka-Kogo A, Kusaka M, Omichi K, Suzuki R, Kato-Fukui Y, Kamiirisa K, Matsuo M, Kamijo S Kasahara M, Yoshioka H, Ogata T, Fukuda T, Kondo I, Kato M, Dobyns WB, Yokoyama M, et al. (2002) Mutation of ARX causes abnormal development of forebrain and testes in mice and X-linked lissencephaly with abnormal genitalia in humans. Nat Genet 32:359-369.

Lehmann J, Nagy JI, Atmadia S, Fibiger HC (1980) The nucleus basalis magnocellularis: the origin of a cholinergic projection to the neocortex of the rat. Neuroscience 5:1161-1174.

Liodis P, Denaxa M, Grigoriou M, Akufo-Addo C, Yanagawa Y, Pachnis V (2007) Lhx6 activity is required for the normal migration and specification of cortical interneuron subtypes. J Neurosci 27:3078-3089.

Marin O, Anderson SA, Rubenstein JL (2000) Origin and molecular specification of striatal interneurons. J Neurosci 20:6063-6076.

Mesulam MM, Mufson EJ, Wainer BH, Levey AI (1983) Central cholinergic pathways in the rat: an overview based on an alternative nomenclature (Ch1-Ch6). Neuroscience 10:1185-1201.

Mori T, Yuxing Z, Takaki H, Takeuchi M, Iseki K, Hagino S, Kitanaka J, Takemura M, Misawa H, Ikawa M, Okabe M, Wanaka A (2004) The LIM homeobox gene, L3/Lhx8, is necessary for proper development of basal forebrain cholinergic neurons. Eur J Neurosci 19:3129-3141.

Pfaff SL, Mendelsohn M, Stewart CL, Edlund T, Jessell TM (1996) Requirement for LIM homeobox gene Isl1 in motor neuron generation reveals a motor neuron-dependent step in interneuron differentiation. Cell 84:309-320.

Shaftel SS, Carlson TJ, Olschowka JA, Kyrkanides S, Matousek SB, O’Banion MK (2007) Chronic interleukin-1 $\beta$ expression in mouse brain leads to leukocyte infiltration and neutrophil-independent blood brain barrier permeability without overt neurodegeneration. J Neurosci 27:9301-9309.

Stenman J, Toresson H, Campbell K (2003) Identification of two distinct progenitor populations in the lateral ganglionic eminence: implications for striatal and olfactory bulb neurogenesis. J Neurosci 23:167-174.

Sussel L, Marin O, Kimura S, Rubenstein JL (1999) Loss of Nkx2.1 homeobox gene function results in a ventral to dorsal molecular respecification within the basal telencephalon: evidence for a transformation of the pallidum into the striatum. Development 126:3359-3370.

Thor S, Ericson J, Brannstrom T, Edlund T (1991) The homeodomain LIM protein Isl-1 is expressed in subsets of neurons and endocrine cells in the adult rat. Neuron 7:881-889.

Thor S, Andersson SG, Tomlinson A, Thomas JB (1999) A LIMhomeodomain combinatorial code for motor-neuron pathway selection. Nature 397:76-80.

Wang HF, Liu FC (2001) Developmental restriction of the LIM homeodomain transcription factor Islet-1 expression to cholinergic neurons in the rat striatum. Neuroscience 103:999-1016.

Whitehouse PJ, Price DL, Clark AW, Coyle JT, DeLong MR (1981) Alzheimer disease: evidence for selective loss of cholinergic neurons in the nucleus basalis. Ann Neurol 10:122-126.

Woolf NJ (1991) Cholinergic systems in mammalian brain and spinal cord. Prog Neurobiol 37:475-524.

Woolf NJ, Eckenstein F, Butcher LL (1983) Cholinergic projections from the basal forebrain to the frontal cortex: a combined fluorescent tracer and immunohistochemical analysis in the rat. Neurosci Lett 40:93-98.

Zhao Y, Marin O, Hermesz E, Powell A, Flames N, Palkovits M, Rubenstein JL, Westphal H (2003) The LIM-homeobox gene Lhx8 is required for the development of many cholinergic neurons in the mouse forebrain. Proc Natl Acad Sci USA 100:9005-9010. 